\title{
Design and Simulation of Voltage Measurement System Based on ADC0809
}

\author{
Huiling Jin, Zhaohan Zhang \\ Zhoukou Vocational and Technical College, Henan, China
}

\begin{abstract}
:
The display of ordinary voltmeter is not clear enough, the measurement range is narrow and the antiinterference ability is weak. This paper studies the design and Simulation of voltage measurement system based on ADC0809. Combined with the actual production situation, using the function that ADC0809 has 8 analog inputs and can convert the analog input into digital display, this paper designs the circuit with single chip microcomputer chip AT89S51. Combined with the working principle of a / D converter and DC digital voltmeter composed of them, the voltage measurement of $0-10 \mathrm{~V}$ is realized in this paper. The experimental results show that the measurement system has the advantages of accurate reading, wide measurement range and micro power consumption..
\end{abstract}

Keywords: ADC0809, Voltage Measurement System, Simulation.

\section{INTRODUCTION}

At present, the safe and stable operation of the power system provides a solid guarantee for the development of the whole national economy [1-2]. As a power distribution hub, the normal operation of various substations is the basis of the health and stability of the whole power grid. The storage battery in the DC system of the substation plays an important role in providing continuous power to all commercial equipment of the whole station, including protection devices and communication equipment, when the $\mathrm{AC}$ power of the primary system of the whole station is lost [3]. Under normal circumstances, the AC power of the primary system can be reduced through the transformer and then through the rectifier module to obtain about $240 \mathrm{~V}$ DC power supply, so as to charge more than a dozen batteries in series [4-6]. However, after a long time of operation, due to the subtle differences such as different internal resistance of each battery, the partial voltage of each battery is uneven, which may lead to uneven charging of each battery. In serious cases, the voltage of each battery varies greatly, and even all batteries are damaged and can not be charged [7]. If the AC umbrella of the primary system loses power at this time, the battery will not play its due role. However, at present, the DC system status of many substations can only be known by the daily patrol of operators, and the remote monitoring 
Article History: Received: 28 October 2021 Revised: 05 December 2021 Accepted: 10 January 2022 Publication: 28 February 2022

and alarm of battery voltage can not be realized, which is obviously a hidden danger.

\section{SCHEME DESIGN OF BATTERY VOLTAGE TELEMETRY SYSTEM}

1.Working principle of substation DC system

The DC system provides $\pm 110 \mathrm{~V}$ DC power supply for the substation and plays a vital role as the power source for all communication equipment, protection and monitoring devices in the whole station. SR-2 dual AC incoming line switching device inputs dual three-phase AC and outputs one. Its function is to take only one of the two AC incoming lines as output when both $\mathrm{AC}$ incoming lines are powered. The other circuit is in standby state, and when the AC incoming line of this circuit loses power, it can automatically switch to the standby incoming line to keep the output continuous. Because there may be phase difference between the two AC incoming lines, the device makes the two incoming lines disconnected from each other during JF constant and switching [8].

The AC power supply is converted into $\pm 110 \mathrm{~V}$ true current power supply through rectifier device (cv1-cv4). After passing through the diode (the function of the diode is to prevent reverse power transmission), the battery is charged and the load is directly supplied. When the battery is charged, KML is closed, RDL and RD2 are two fuses, and their auxiliary contacts can be used to monitor whether they operate well. GB is a battery. Generally, a single battery with rated $12 \mathrm{~V}$ is used, which is usually charged to about $13.4 \mathrm{v}$ to ensure sufficient capacity when the battery is discharged. Therefore, the voltage of DC bus + HM is more than ten volts higher than that of $+\mathrm{km}$, and the load is finally supplied by $+\mathrm{km}$ and one $\mathrm{M}$. the step-down device from $+\mathrm{HM}$ to $+\mathrm{km}$ is sa. The change-over switch can control which diodes are turned on, so as to realize the step-down function.

\section{Scheme design of battery voltage telemetry system}

There are two schemes to realize the monitoring of each battery. One is to use the existing DC system, increase the voltage measurement function, and try to send the voltage information to the remote background. This method is difficult to realize. Firstly, the computer system resources of the original DC monitoring device shall be increased, and the DC system manufacturer shall open the communication interface and increase the corresponding soft functions. In addition, at present, the DC system of the substation has no communication function with the background of the station, and the signal is completely realized by the hard open contact, so this method is not feasible.

Another scheme is enough to add a voltage acquisition system completely independent of the existing DC system, which is completely independent from device power supply to data communication. It can collect voltage and upload it to the remote background without affecting the operation of the existing DC system. I chose the latter.

The A/D conversion module is controlled by single chip microcomputer to measure the voltage. After data processing, it is uploaded to the background computer for analysis to realize 
Article History: Received: 28 October 2021 Revised: 05 December 2021 Accepted: 10 January 2022 Publication: 28 February 2022

the voltage display and alarm function. Figure 1 shows the circuit design scheme of the voltage acquisition system.

The battery pack is generally composed of 18 or 36 single cells in series. Some substations are equipped with two sets of batteries. It is impossible to collect all battery voltage information by using an aid conversion module. This faces two choices. One single chip microcomputer can be used with multiple aid conversion modules, or one single chip microcomputer can be used with one A/D conversion module, and then multiple single chip microcomputer systems can complete the collection of battery voltage.

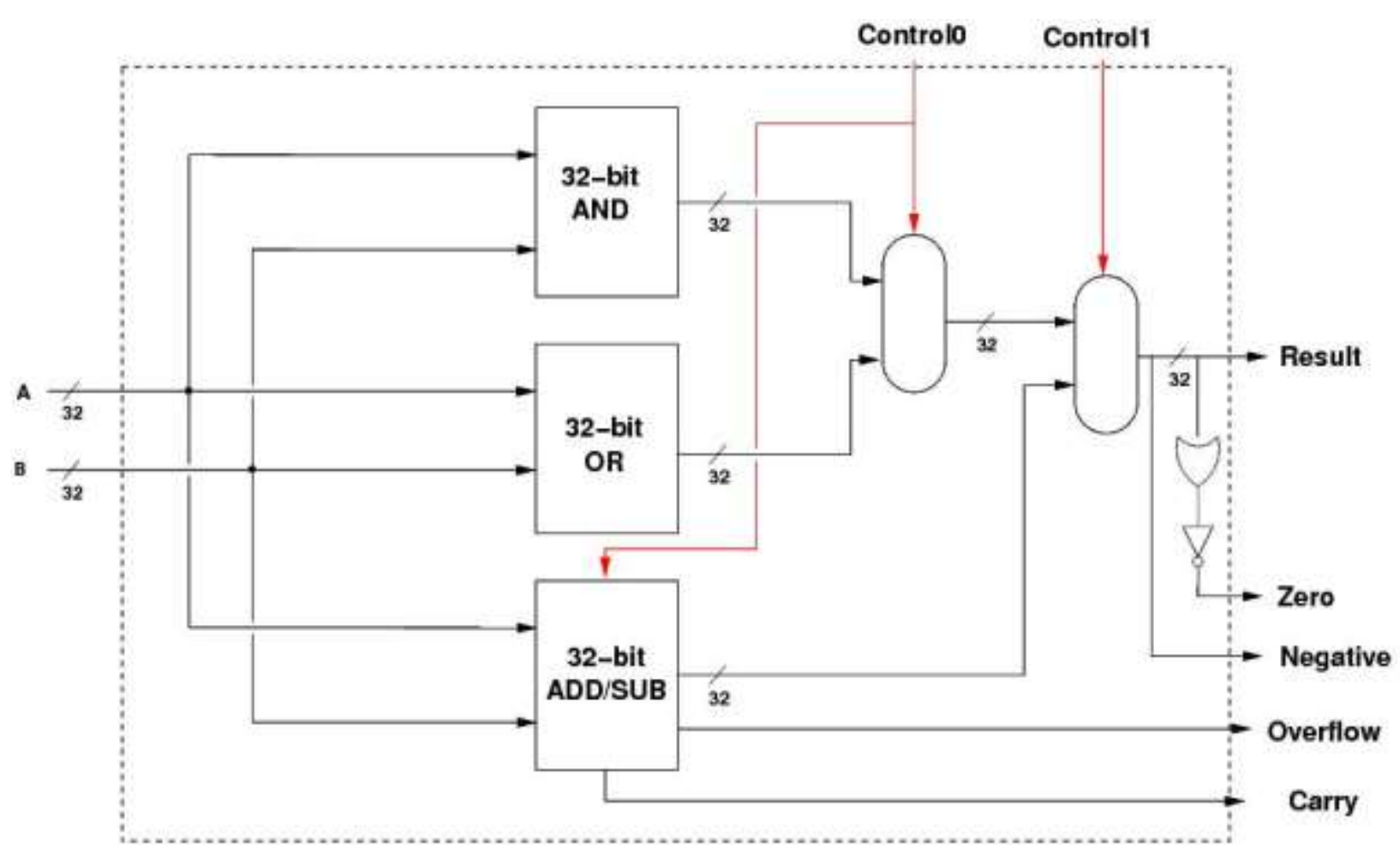

Fig 1: Circuit design scheme of battery voltage acquisition system 
Article History: Received: 28 October 2021 Revised: 05 December 2021 Accepted: 10 January 2022 Publication: 28 February 2022

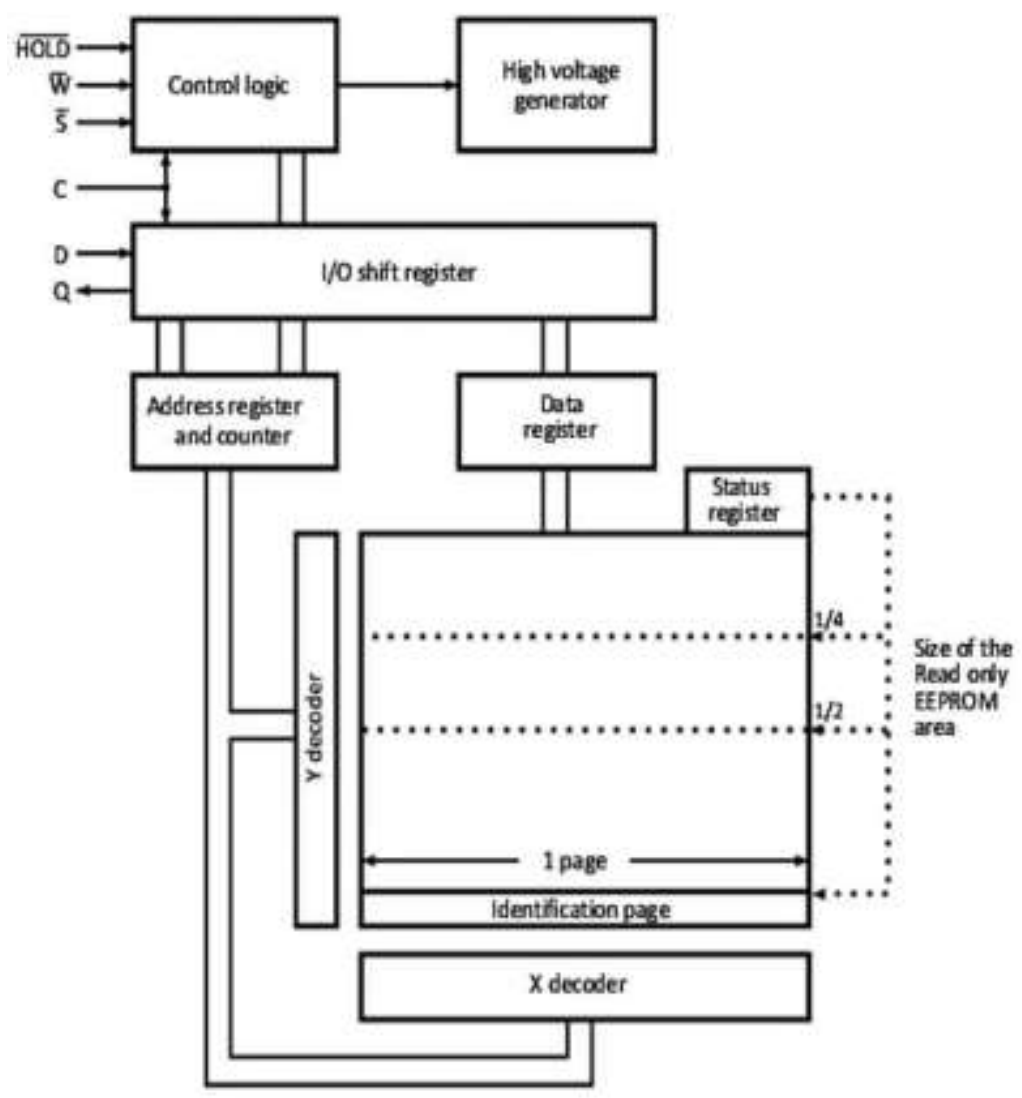

Fig 2: Schematic diagram of a single chip microcomputer multi A / D conversion module

\section{COMPILATION AND IMPLEMENTATION OF COMMUNICATION PROTOCOL FOR VOLTAGE TELEMETRY SYSTEM}

The voltage telemetry system is composed of a PC computer as the upper computer and multiple single chip computers as the lower computer. Therefore, each single chip microcomputer should have its own address information to facilitate control and identification. First, the PC sends the read voltage command to a single chip microcomputer. Each single chip microcomputer receives the command and determines whether the command is sent to itself. If so, the single chip microcomputer starts to work, controls the A/D conversion module to measure the voltage of each battery connected to it, and sends it one by one to the PC. after the single chip microcomputer is sent, the PC machine sends the voltage reading command to another single chip microcomputer again, and circulates in turn until all the voltage information is read.

The system has poor timeliness requirements, so asynchronous communication should be adopted; The amount of information is small and the communication speed is not high, but each 
Article History: Received: 28 October 2021 Revised: 05 December 2021 Accepted: 10 January 2022 Publication: 28 February 2022

frame should contain MCU address and battery voltage data, so each frame should be composed of multiple bytes. In addition, from the function of the system, the single chip microcomputer only receives instructions from the $\mathrm{PC}$, and the master-slave question and answer communication can be adopted.

PC machine first obtains an address from the MCU address list, selects the read voltage command and sends a data frame. Only the specified MCU starts to work. Because the A / D module has multiple addresses, that is, multiple batteries are connected, the single chip microcomputer sends a data frame to the PC machine every time it reads a battery voltage from the A / D module. The data frame contains voltage data, which is the address information of the battery and the single chip microcomputer. Then the MCU continues to read the next battery voltage data and send it out until all connected battery voltages are read. At this time, send the voltage reading data frame to the $\mathrm{PC}$, and the $\mathrm{PC}$ obtains an address from the address list and sends the voltage reading instruction. Repeat the above until the PC sends all the addresses.

In view of the communication requirements of the voltage telemetry system, the system realizes the question and answer communication between the master (PC) and the slave (single chip microcomputer). The PC is always the master controller, and the single chip microcomputer is only the passive receiver. Therefore, the data packets received or sent by PC and MCU can be different. This application adopts an indefinite length data frame composed of multiple bytes. Table 1 shows the command response table of communication between PC and MCU.

\section{TABLE I. Comparison table of PC instruction slave response}

\begin{tabular}{|c|c|c|c|}
\hline $\begin{array}{c}\text { Serial } \\
\text { numbe } \\
\text { r }\end{array}$ & $\begin{array}{c}\text { Host } \\
\text { command }\end{array}$ & Slave response & Slave response comment \\
\hline 1 & AAaddr0155 & AA01addr55 & $\begin{array}{c}\text { 01 is the command to view the device } \\
\text { address, and addr is the slave address }\end{array}$ \\
\hline 2 & AAaddr0255 & AA02addr55 & $\begin{array}{c}02 \text { is the command to stop transmitting } \\
\text { data, addr is the slave address, and the } \\
\text { response indicates that the slave has } \\
\text { stopped sending data to the host }\end{array}$ \\
\hline 3 & AAaddr0355 & $\begin{array}{c}\text { AA03addrDataParam00 } \\
\text { is } 5\end{array}$ & $\begin{array}{c}\text { 03 is to start sending data command, addr } \\
\text { is the slave address, data is the 8-bit data } \\
\text { of analog voltage after A / D conversion, } \\
\text { and param represents the battery } \\
\text { connected to the single chip } \\
\text { microcomputer }\end{array}$ \\
\hline
\end{tabular}


Article History: Received: 28 October 2021 Revised: 05 December 2021 Accepted: 10 January 2022 Publication: 28

\begin{tabular}{|c|c|c|c|} 
& AA03addrData0055 & $\begin{array}{c}\text { Setting param to zero indicates that all } \\
\text { battery voltages connected to the single } \\
\text { chip microcomputer have been } \\
\text { transmitted }\end{array}$ \\
\hline 4 & AAaddr0455 & AA04addr55 & $\begin{array}{c}04 \text { is the command to verify the } \\
\text { connection status, and addr is the slave } \\
\text { address. The response indicates that the } \\
\text { slave is in good connection with the host }\end{array}$ \\
\hline
\end{tabular}

\section{DESIGN AND IMPLEMENTATION OF HARDWARE CIRCUIT}

As a basic circuit acquisition system, there should be the acquisition module with the highest voltage and the processing module for the collected data. Finally, the data should be transmitted to the PC for processing, analysis, display and alarm. Therefore, there should be a communication module in the middle to realize the communication between the data acquisition and processing module and the PC. As shown in Figure 3, this is actually a common PC single chip microcomputer system, a widely used master-slave computer control system. 
Article History: Received: 28 October 2021 Revised: 05 December 2021 Accepted: 10 January 2022 Publication: 28 February 2022

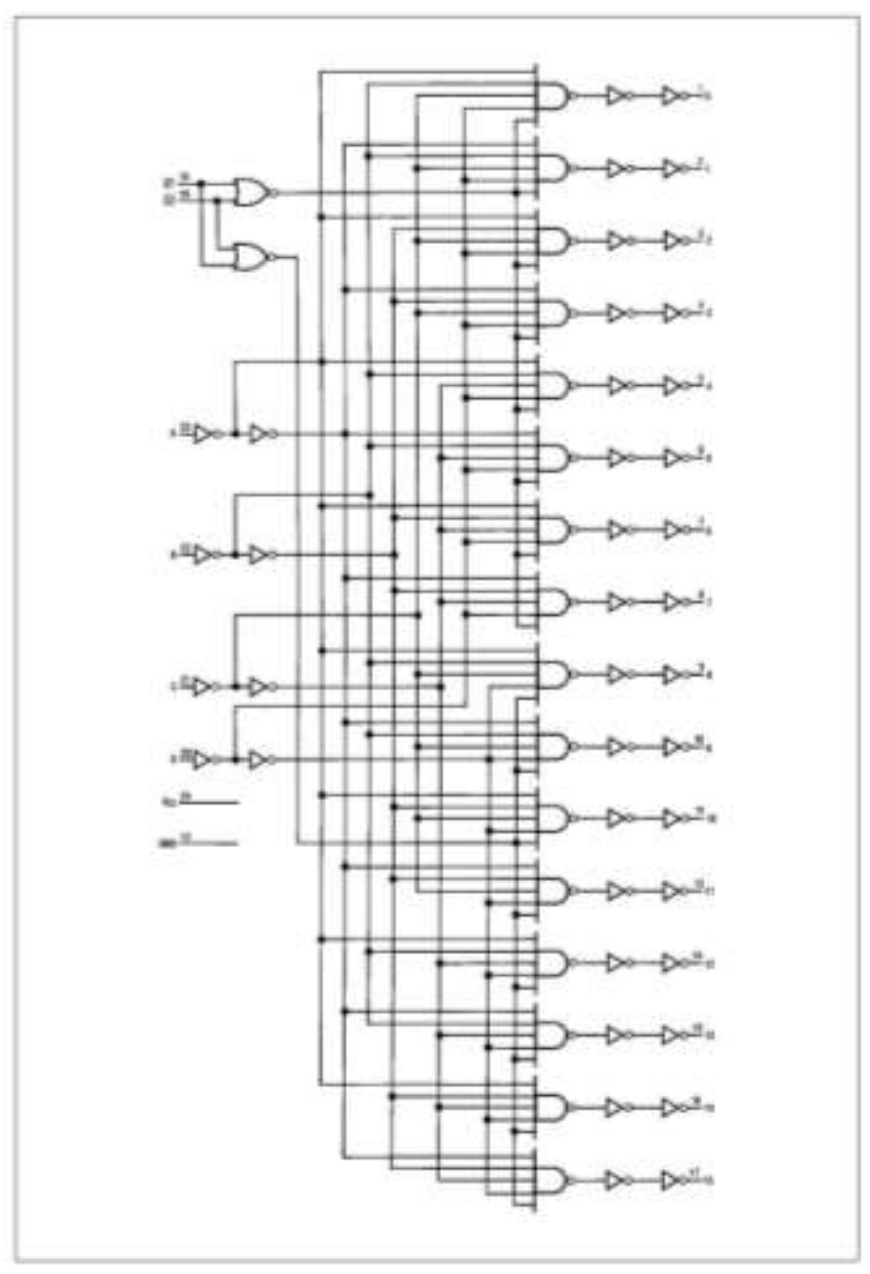

Fig 3: Logic diagram of PC single chip microcomputer system

The realization of serial communication needs level conversion circuit. The level conversion circuit adopts max232cpe produced by Maxim company to complete the mutual conversion between PC serial port voltage and MCU serial port voltage. The differences between the two are briefly introduced below.

First, let's look at the RS-232C standard. The full name of RS232C standard is eia.rs-232c standard, in which EIA (Electronic Industry Association) represents the American Electronic Industry Association. RS (recommended standard) stands for the recommended standard, 232 is the identification number, and C stands for the latest modification of RS232. Before that, there were rs232b and rs232a. It is a standard for serial communication jointly formulated by the American Electronics Industry Association and bell system, modem manufacturers and computer terminal manufacturers in 1969. Its umbrella name is "technical standard for serial 
Article History: Received: 28 October 2021 Revised: 05 December 2021 Accepted: 10 January 2022 Publication: 28 February 2022

binary data exchange interface between data terminal equipment (DTE) and data communication equipment (DCE)". At present, the coml and com2 interfaces on general PC are RS-232C interfaces.

In addition, the "send" and "receive" mentioned in RS232C standard are defined from the standpoint of DTE rather than DCE. In computer systems, information is often transmitted between CPU and I / O devices, both of which are DTE, so both sides can send and receive.

Finally, the communication allowable distance of RS-232C standard is briefly described. When there is no modem, the maximum communication distance is calculated as follows: when the bit error rate is less than $4 \%$, the capacitance of the conductor shall be less than 2500pf. For ordinary wires, if the capacitance is about $170 \mathrm{pf} / \mathrm{m}$, the allowable distance $\mathrm{L}=2500 \mathrm{pf} / 170$ $(\mathrm{PF} / \mathrm{M})=15 \mathrm{~m}$. The calculation of this distance is conservative. In practical application, when $9600 \mathrm{bps}$ and ordinary twisted pair shielded wire are used, the distance can be up to $30 \mathrm{~m}-35 \mathrm{~m}$. The computer used in the system is the background machine in the substation, and the distance meets the above requirements.

The A / D conversion module adopts ADC0809 circuit, which is a typical 8-bit 8-channel successive approximation A / D converter with CMOS structure. Adc0809 is a typical device commonly used in the application of single chip microcomputer. Its circuit connection and function are very mature and easy to use. The resolution of ADC0809 is 8 bits. Because the functional composition of this system is very simple, its single chip microcomputer will select 8 bits, so it is enough to select ADC0809 with 8 data bits. The conversion time of ADC0809 depends on the chip clock frequency. The conversion time is 64 clock cycles. When CLK = $500 \mathrm{KHz}$, the conversion time is $\mathrm{T}=128 \mu \mathrm{s}$. Because the real-time requirement of the system is not high, and the interrupt processing mode is adopted, there will be no speed mismatch and timing mismatch. Adc0809 adopts single power supply $+5 \mathrm{~V}$, which is the same as that of MCU. The analog input voltage range is unipolar $0+5 \mathrm{~V}$, while the general battery voltage is about $12 \mathrm{~V}$, which is greater than $+5 \mathrm{~V}$. Therefore, a simple step-down treatment should be done at the analog input interface. Adc0809 has a controllable three state buffer output latch, which can be directly connected with the data line of a single chip microcomputer. Adc0809 also has some functional control lines, which can realize message exchange with the outside world and accept control.

The in system programming (ISP) function enables users to program without removing the single chip microcomputer from the system. The ISP feature is realized by a series of hardware resources in the single chip microcomputer. The core is equipped with an internal firmware, which can realize the online programming of P89V51RD2 through the serial port. The firmware is provided by Philips and embedded in the reel.

P89V51RD2FN on-chip program memory adopts the most advanced FLASH EPROM memory structure, which has $64 \mathrm{~K}$ and is divided into $8 \mathrm{~K}$ and $16 \mathrm{~K}$ memory blocks. The improvement of FLASH EPROM memory over EPROM memory is that it can not only be read, 
Article History: Received: 28 October 2021 Revised: 05 December 2021 Accepted: 10 January 2022 Publication: 28 February 2022

but also be quickly erased and written by software, thus realizing the new technology of ISP.

Philips Company provided a 1kbyte firmware called BootROM for P89V51RD2FN on chip. The firmware includes boot loader, which can receive commands and data from the host through serial port (such as RS232 port of PC), and various subroutines such as serial erasing and writing of FLASH. This piece is placed at the highest end of $64 \mathrm{~K}$ program memory, which is covered with the on-chip FLASH address 0FC00H-0FFFFH.

\section{CONCLUSION}

As a standby DC power supply, the battery pack in the substation DC system plays an important role in providing DC power when the AC power of the whole station is lost. However, how to monitor the voltage information of the battery and understand the operation status of the battery has been perplexing the operation personnel on duty in some substations. This paper designs and implements a battery voltage real-time acquisition system. It is worth mentioning that the system is separated from the original DC system and completely independent of the original communication system, which increases the reliability of operation. In addition, the whole hardware cost of the system is very low, which fully meets the needs of power operation without great transformation of substation DC system.

\section{ACKNOWLEDGEMENTS}

This research is supported by the key scientific research projects of colleges and universities in Henan Province (Grant No. 20B413011.). This study was supported by the science and technology public relations project of Henan Province (Grant No. 192102210250)

\section{REFERENCES}

[1] Wong, L. H., Chai, C. S., Zhang, X., \& King, R. B., (2015). Employing the tpack framework for researcher-teacher co-design of a mobile-assisted seamless language learning environment. IEEE Transactions on Learning Technologies, 8(1), 31-42.

[2] Kim, H., Shin, H., Kim, H. S., \& Kim, W. T., (2018). Vr-cpes: a novel cyber-physical education systems for interactive vr services based on a mobile platform. Mobile Information Systems, 2018(PT.3), 1-10.

[3] Ji, X., (2019). Community guidance model based on interactive multimedia system. Multimedia Tools and Applications, 78(4), 4723-4741.

[4] Chang, C. K., Reisman, S., \& Tovar, E., (2017). Advances in learning technologies. Computer, 50(5), 14-17. 
Article History: Received: 28 October 2021 Revised: 05 December 2021 Accepted: 10 January 2022 Publication: 28 February 2022

[5] Chacon, R., Codony, D., \& Toledo, A., (2017). From physical to digital in structural engineering classrooms using digital fabrication. Computer Applications in Engineering Education, 25(6), 927937.

[6] Ahmed, \& Ali, A., (2015). A comparative study of qos performance for location based and corona based real-time routing protocol in mobile wireless sensor networks. Wireless Networks, 21(3), 1015-1031.

[7] Friedman, A., Hahn, K. A., Etz, R., Rehwinkel-Morfe, A. M., Miller, W. L., \& Nutting, P. A., et al. (2014). A typology of primary care workforce innovations in the united states since 2000. Medical Care, 52(2), 101.

[8] Premalatha M (2008) Efficient cogeneration scheme for sugar industry. Journal of Scientific \& Industrial Research 67:239-242 